\title{
X-ray imaging with compound refractive lens and microfocus $X$-ray tube
}

\author{
Ladislav Pina ${ }^{1 *}$, Yury Dudchik ${ }^{2}$, Vaclav Jelinek ${ }^{3}$ Libor Sveda $^{1}$, Jiri Marsik ${ }^{3}$, Martin Horvath $^{3}$ \\ Ondrej Petr ${ }^{3}$, \\ ${ }^{1}$ Czech Technical University in Prague, V. Holesovickach 2, 18000 Prague 8, Czech Republic \\ ${ }^{2}$ Institute of Applied Physics Problems of Belarus State University, Kurchatova 7, 220064 Minsk, Belarus \\ ${ }^{3}$ Reflex s.r.o., Novodvorska 994, 14221 Prague 4, Czech Republic
}

\begin{abstract}
Compound refractive lenses (CRL), consisting of a lot number in-line concave microlenses made of low-Z material were studied. Lenses with focal length $109 \mathrm{~mm}$ and $41 \mathrm{~mm}$ for 8-keV X-rays, microfocus X-ray tube and X-ray CCD camera were used in experiments. Obtained images show intensity distribution of magnified microfocus X-ray source focal spot. Within the experiments, one lens was also used as an objective lens of the X-ray microscope, where the copper anode X-ray microfocus tube served as a source. Magnified images of gold mesh with 5 microns bars were obtained. Theoretical limits of CRL and experimental results are discussed.
\end{abstract}

Keywords: X-ray optics, compound refractive lenses, X-ray imaging.

\section{INTRODUCTION}

Compound refractive lens (CRL), consisting of a lot number of placed in-line concave microlenses made of low-Z material, is a unique imaging X-ray device ${ }^{1-3}$. The lens focal length $f$ is proportional to the individual lens curvature radius $R$ and inversely proportional to the number of microlenses $N$ :

$$
f=\frac{R}{2 N \delta},
$$

where $(1-\delta)$ is real parts of refractive index $n$, where $n=1-\delta-i \beta$, and $\beta$ is an imaginary part. For example, focal length of the lens consisting of 150 epoxy microlenses with 50 microns curvature radius each is approximately equal to $45 \mathrm{~mm}$ for 8-keV.

Compound refractive lenses (CRL) may be produced by different methods: by pressing individual lenses followed by alignment ${ }^{4}$, LIGA method ${ }^{5}$, and lithographic technique ${ }^{6}$. The lenses also may be designed by the way described in ${ }^{7-}$ 9 , where a set of spherical concave microlenses with curvature radius $R$ was formed inside glass capillary with the same radius $\mathrm{R}$. The microlenses inside the capillary were formed by putting air bubbles into epoxy. It was found that the microlenses have spherical shape and its curvature radius $R$ is equal to the capillary one ${ }^{9}$. The lenses are compact and may be formed inside glass capillaries with inner diameter up to 20-50 microns. In this case expected lens curvature radius will be equal to $10-25$ microns and such lenses are difficult to produce by another methods.

Experiments with synchrotron $8 \mathrm{keV}$ and $18 \mathrm{keV}$ X-rays at SSRL and APS ${ }^{10,11}$ shown that the lenses formed inside glass capillary really focuses radiation into $1-2$ microns spots. Previously it also was shown that the lenses due to its short focal length are suitable for imaging experiments with conventional X-ray tube as a source of radiation ${ }^{12-14}$.

Here we describe using CRLs for imaging of the focal spot of microfocus X-ray tube (Reflex, $40 \mathrm{kV}, 2 \mathrm{~mA}, 60$ microns spot size). Two lenses with focal length $109 \mathrm{~mm}$ (lens \#1) and $41 \mathrm{~mm}$ (lens \#2) for $8 \mathrm{keV}$ X-rays were used for experiments. Spherical microlenses were produced inside glass capillary with diameter 200 microns and 100 microns for lens \#1 and \#2 respectively by the method described in ${ }^{7-9}$. Lens \#2 also was used as objective lens for a simple X-ray

\footnotetext{
*Correspondence E-mail: ladislav.pina@fjfi.cvut.cz
} 
microscope. The copper anode X-ray tube was used as a source and 8 times increased image of gold mesh with 5microns wires was obtained.

\section{IMAGING X-RAY TUBE FOCAL SPOT}

Compound refractive X-ray lens is imaging device and it works like ordinary lens for visual light. It means that if we put a lens at a distance $a$ to the X-ray source, the lens forms source image at a distance $b$, as it is shown in Fig. 1 , in accordance with a well-known lens formula:

$$
\frac{1}{a}+\frac{1}{b}=\frac{1}{f}
$$

Formula (2) is valid for a thin lens. For a thick lens the formula should be corrected by the way proposed, for example, in ${ }^{15}$, where it is shown that for a thick lens distances $a$ and $b$ are measured from lens principal axis.

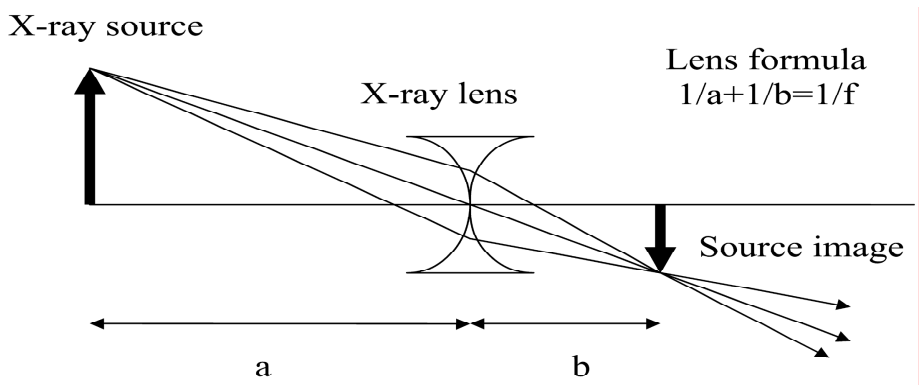

Fig.1. Paths of X-rays forming X-ray source image.

The X-ray lenses for experiments on imaging were designed in the Institute of Applied Physics Problems (Belarus) by putting air bubbles inside a capillary prefilled by epoxy as is described in ${ }^{7-9}$. Fig. 2 is a photo of glass capillary with diameter 200 microns filled by a set of microlenses. The air bubbles between the lenses correspond to black areas.

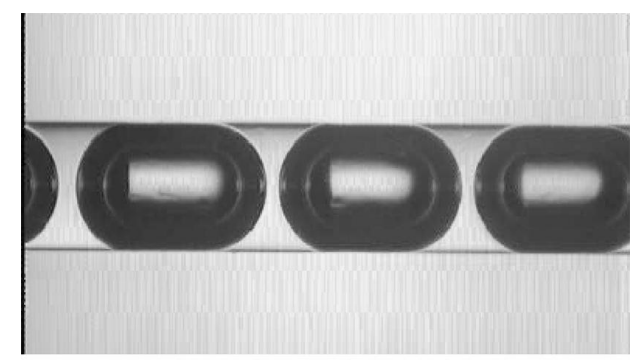

Fig. 2. Photo of the capillary filled by epoxy concave microlenses. Capillary diameter is equal to 200 microns.

Two lenses were used for imaging. The lens \#1 consists of 121 concave spherical epoxy microlenses, each with curvature radius equal to 100 microns. The microlenses were formed inside capillary with inner diameter equals to 200 microns. Lens \#1 length is equal to $38 \mathrm{~mm}$. The lens \#2 consists of 161 concave spherical epoxy microlenses, each with curvature radius equal to 50 microns. The microlenses were formed inside capillary with inner diameter equals to 100 micron. Lens \#2 length is equal to $18 \mathrm{~mm}$. The lens focal length $f$ was calculated by formula (2) with parameter $\delta$, that was obtained from next equation :

$$
\delta=0.5\left(\frac{22}{E}\right)^{2}
$$


where $E$ is photon energy measured in $\mathrm{eV}$. The value $22 \mathrm{eV}$ is plasmon energy that was calculated if the lens material (epoxy) was considered in the context of free electron gas theory. Used epoxy consists of carbon, oxygen, hydrogen and nitrogen which are chemically bonded in proportion $\mathrm{C}_{200} \mathrm{H}_{100} \mathrm{O}_{20} \mathrm{~N}$. The epoxy density is $1.08 \mathrm{~g} / \mathrm{cc}$. Result for calculation of the lens focal length for $8 \mathrm{keV} \mathrm{X}$-rays is: $f=109 \mathrm{~mm}$ for lens \# 1 and $\mathrm{f}=41 \mathrm{~mm}$ for lens \# 2 .

The lens \#1 and lens \#2 were used for imaging X-ray tube focal spot. X-ray CCD camera was used to imagine Xray intensity distribution. X-ray tube, X-ray lens and CCD camera were placed in line at a distances $a$ and $b$ to each another that satisfy to a lens formula (2). a microfocus X-ray tube with copper anode was used as a source of X-rays. The size of the X-ray tube focal spot is equal approximately to 50 microns at $40 \mathrm{kV}$ voltages. The X-ray tube was placed at a different distances to the lens and the source image was recorded by the X-ray cameras. Two X-ray cameras were used: one is the fast X-ray digital CCD camera (RX 1C, Reflex) with GADOX $\operatorname{Gd}_{2} \mathrm{O}_{2} \mathrm{~S}$ scintillator, active area is $14.5 \mathrm{x}$ $10.9 \mathrm{~mm}^{2}, 23.5 \mu \mathrm{m} \times 23.5 \mu \mathrm{m}$ effective pixel size, spectral range from 5 to $20 \mathrm{keV}$. The other one is X-ray sub-micron resolution CCD camera (Reflex) with LuAG: Ce scintillator (active area is $1.02 \times 0.83 \mathrm{~mm}, 0.645 \mu \mathrm{m} \times 0.645 \mu \mathrm{m}$ effective pixel size, spectral range from 0.05 to $50 \mathrm{keV}$ ).

The size of the source image $S_{1}$ depends on the distance $a$ between the source and the lens and may be calculated as $S_{I}=S M$, where $S$ is source size, $M$ - magnification. The magnification $M$ depends on $a$ and $b$ as $M=b / a$, where b is distance from the lens to the source image. For the case when $a=b=2 f$ the size of the image is equal to the source size.

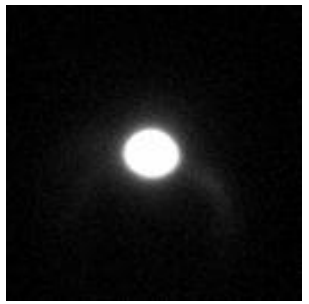

a)

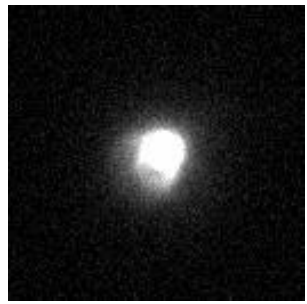

b)

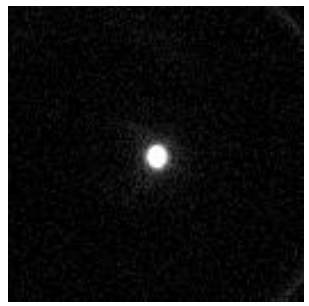

c)

Fig.3. Images of X-ray tube focal spot captured by the X-ray digital CCD camera with lens\#1 at different magnification $\mathrm{M}$ : a) $\mathrm{M}=2.7$ ;b) $\mathrm{M}=1$; c) $\mathrm{M}=0.32$.

Fig. 3 Shows images of X-ray tube focal spot captured by X-ray digital CCD camera (RX 1C) with lens\#1 at different magnification $M$ : a) $a=150 \mathrm{~mm}, b=400 \mathrm{~mm}, M=2.7$;b) $a=218 \mathrm{~mm}, b=218 \mathrm{~mm}, M=1$; c) $a=450 \mathrm{~mm}, b=144$ $\mathrm{mm}, M=0.32$. Tube voltage $=40 \mathrm{kV}$, current $=1 \mathrm{~mA}$, exposition was equal to $0.2 \mathrm{~s}$.

Fig. 4 Shows demagnified X-ray tube focal spot image taken with lens \#2 and the X-ray micron resolution CCD camera. Distances $a$ and $b$ were equal to $250 \mathrm{~mm}$ and $47 \mathrm{~mm}$ respectively. Tube voltage $=40 \mathrm{kV}$, current $=2 \mathrm{~mA}$, exposure $=900 \mathrm{~s}$. The image of the tube focal spot in Fig. 4 is much more informative than image shown in Fig. 3 because the spatial resolution of used X-ray CCD camera is less than 1 micron.

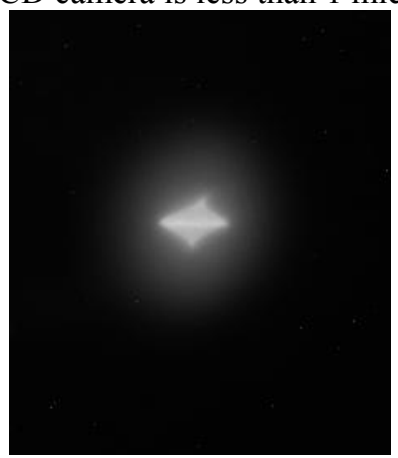

Fig. 4. Demagnified $(M=0.2) X$-ray tube focal spot image taken with lens \#2 and the X-ray micron resolution CCD camera.

Lens \#1 and fast X-ray digital CCD camera RX 1C were used to take image of X-ray tube focal spot at different applied voltages. Results are shown in Fig. 5. The images were taken at magnification $\mathrm{M}=1$. 


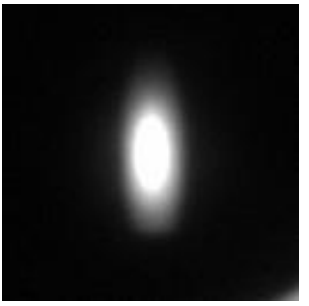

a)

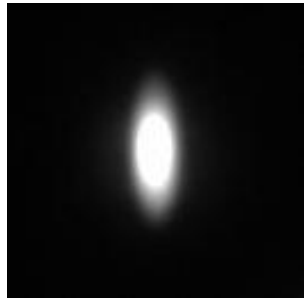

b)

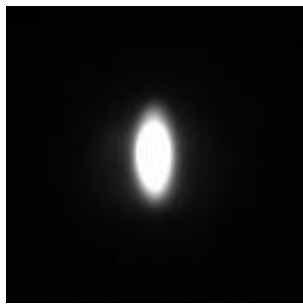

c)

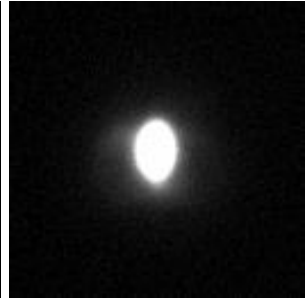

d)

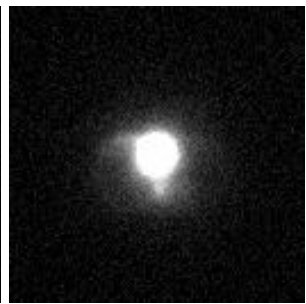

e)

Fig. 5. Images of X-ray tube focal spot at a different tube voltages: a) $U=32 \mathrm{kV}, \mathrm{b}) \mathrm{U}=34 \mathrm{kV}, \mathrm{c}) \mathrm{U}=36 \mathrm{kV}, \mathrm{d}) \mathrm{U}=38 \mathrm{kV}$, e) $\mathrm{U}=40 \mathrm{kV}$.

\section{X-RAY MICROSCOPY WITH REFRACTIVE LENS}

Lens \#2 with $41 \mathrm{~mm}$ focal length for $8 \mathrm{keV}$ X-rays was used as objective for a simple X-ray microscope. The scheme of the microscope is shown in Fig.6. The microscope consists of the microfocus X-ray tube, X-ray lens and CCD camera. In contrast to results, described previously in ${ }^{12-14}$, here we put X-ray tube far away from the lens. In this case the images of X-ray source and of the object may be found at a different distanced to the lens as it is shown in Fig. 6.

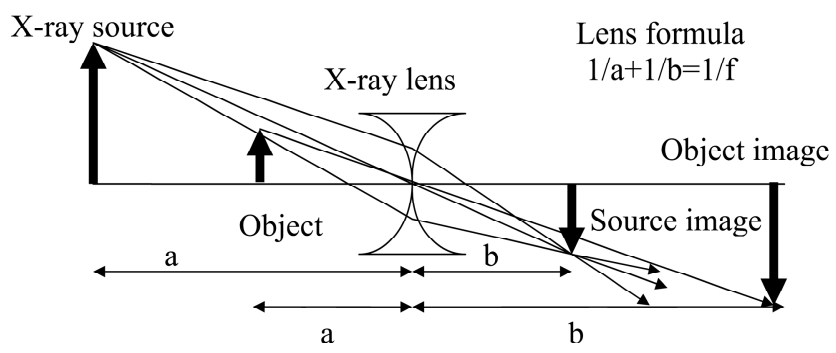

Fig.6. Scheme of a simple X-ray microscope.

Lens \#2 was placed at a distance $185 \mathrm{~mm}$ to $\mathrm{X}$-ray tube. The X-ray tube voltage was chosen equal to $32 \mathrm{kV}$ in order to increase tube focal spot and to decrease bremstrahlung. X-ray tube current was $2 \mathrm{~mA}$. At a given voltage the size of the tube focal spot, as can be seen from Fig 5(a), is more than 100 microns in vertical direction.

Gold mesh \#1000 was used as object for imaging. The mesh \#1000 consists of 5 microns thick wires, with a wire center to center separation of 18 microns. Fast X-ray digital CCD Camera (RX 1C) was used to capture mesh image. The camera was placed at distance $b=405 \mathrm{~mm}$ to the lens. Expected distance $a$ from the object to the lens (calculated with formula 2) is equal to $45.6 \mathrm{~mm}$. In experiment the mesh was moved around $a=46 \mathrm{~mm}$ to achieve more contrast image of the mesh. It was found that the best image is observed when a $=50 \mathrm{~mm}$. The magnification of the microscope is equal to $a / b=8$. Fig. 7 shows images taken by X-ray camera $(\mathrm{a}, \mathrm{b})$ and a result of image computer processing (c). Exposition time is equal to $25 \mathrm{~s}$. Fig. 7 (a) is image of X-ray beam formed by the lens at a distance to lens $b$ equals to $405 \mathrm{~mm}$ when the object is absent. Fig. 7 (b) is image of the mesh $\# 1000$. The mesh was placed at a $=50 \mathrm{~mm}$ to lens and the camera was placed at distance $b=405 \mathrm{~mm}$, as mentioned above. Fig.7 (c) is result of subtraction of two above pictures. As it can be seen from Fig.7(c), 5-microns gold wires are recognized by the camera and we may conclude that the spatial resolution of the discussed $\mathrm{X}$-ray microscope is not worse than 5 microns. 


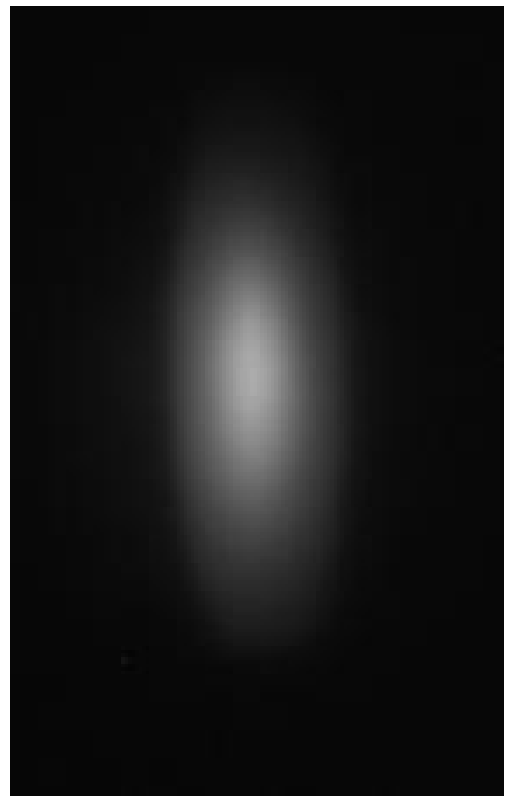

a)

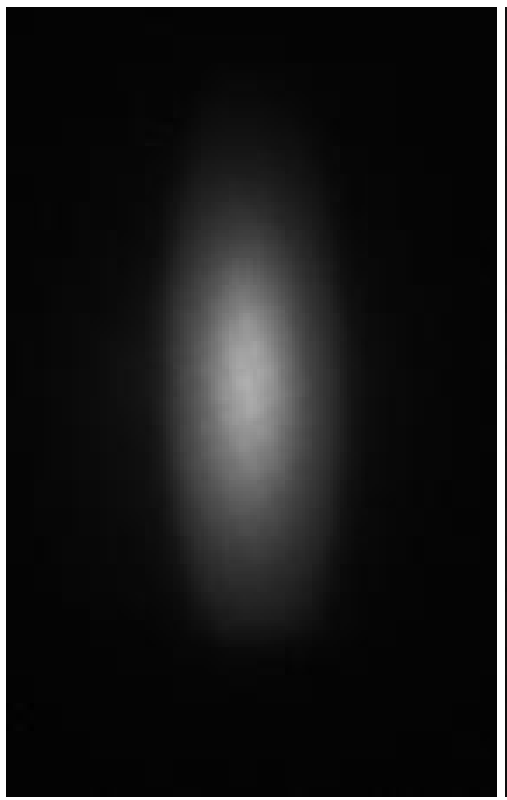

b)

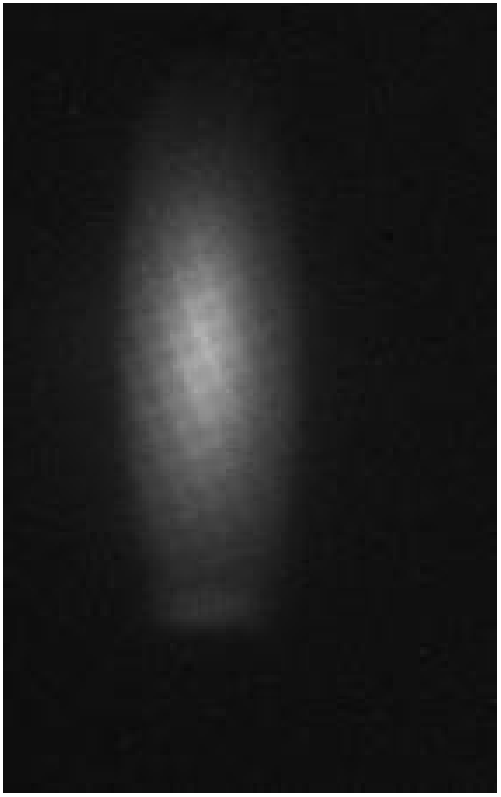

c)

Fig. 7. Images of a) X-ray beam formed by the lens when the object is absent; (b) mesh \#1000. (c) - result of subtraction of image (a) and image (b).

\section{DISCUSSION}

The resolution of the microscope depends on lens parameters, lens quality, magnification factor of the microscope and a spatial resolution of the camera. The spatial resolution of the RX 1C camera is approximately equal to the size of the effective pixel that is equal to $23.5 \mu \mathrm{m} \times 23.5 \mu \mathrm{m}$. The magnification of X-ray microscope is equal to 8 and it is suitable to recognize 3 microns in size objects by using the above mentioned camera. The used lens is spherical one and its resolution is limited by spherical aberrations. Previously it was shown ${ }^{8}$ that spherical compound X-ray lens focuses a beam from a point X-ray source, located at infinity, into a circle with radius $R_{m s}$ defined as:

$$
R m s=\frac{1}{8} \frac{R^{3}}{{ }_{R}^{2}},
$$

where $R_{d}$ is radius of used diaphragm. The lens aperture is restricted by natural diaphragm that is formed due to absorption of X-rays in material of the lens. The lens absorption aperture radius $R_{a}$ in a good approximation can be calculated as:

$$
R_{a}=\left(\frac{2 R}{\mu N}\right)^{\frac{1}{2}}
$$

where $\mu$ is the linear absorption coefficient for the lens material. Calculations shown that $\mathrm{R}_{\mathrm{a}}=32$ microns for lens $\# 2$ at $8 \mathrm{keV}$. In accordance with the formula (4) the lens \#2 may focuses $8 \mathrm{keV} \mathrm{X-rays} \mathrm{into} \mathrm{a} \mathrm{spot} \mathrm{which} \mathrm{diameter} \mathrm{equals} 3.2$ microns. The distribution of the $\mathrm{X}$-rays inside the spot has a pronounced peak around a circle with radius $R_{\text {diff }}$ equals to diffraction limited radius which is approximated by the radius of the first minimum of the Airy diffraction pattern in the focal plane and defined as

$$
R \operatorname{diff}=\frac{0.61 \lambda f}{R d}
$$


where $\lambda$ is wavelength. Calculation shown that $R_{\text {diff }}=0.1$ microns and the lens resolution power may be much better that ones expected from aberration calculations.

To test lens \#2 quality and its spatial resolution we used it for taking a demagnified image of gold mesh \# 1000 . X-ray micron resolution CCD camera was used to captured mesh image. The lens was placed at a distance $250 \mathrm{~mm}$ to $\mathrm{X}$-ray tube focal spot. The mesh \#1000 was placed at a distance $15 \mathrm{~mm}$ to X-ray source. The distance $a$ from the mesh to lens was equal to $235 \mathrm{~mm}$. The position of CCD camera was calculated from the lens formula 2 as $b=49.6 \mathrm{~mm}$. In experiment the camera was placed at $b=47 \mathrm{~mm}$ to achieve better image quality. Tube voltage $=40 \mathrm{kV}$, current $=2 \mathrm{~mA}$, exposure is equal to $900 \mathrm{~s}$.

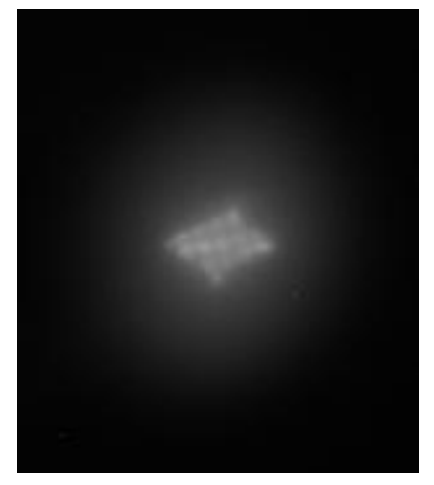

Fig. 8.5 times demagnified X-ray image of mesh \#1000 taken with lens \#2.

Fig. 8 shows 5 times demagnified X-ray image of mesh \#1000 taken with lens \#2. The demagnification was calculated as $\mathrm{a} / \mathrm{b}$, where $\mathrm{a}=235 \mathrm{~mm}$ is distance from mesh to lens, $\mathrm{b}=47 \mathrm{~mm}$ is distance from lens to mesh image. As it can be seen from Fig. 8,5 microns gold wires are clearly resolved by the camera. In accordance with geometry of experiment it is expected that the effective size of the image of 5 microns gold wire is equal to 1 micron. The wires are clearly recognized in Fig. 8 and we may conclude that the lens spatial resolution is not worse than 1 micron.

\section{CONCLUSION}

Experiments have shown that spherical compound refractive X-ray lenses are suitable for imaging in combination with microfocus X-ray tube as a source of radiation. The resolution of the imaging system consisting of X-ray tube, lens and CCD camera depends on lens parameters, lens quality, magnification factor of the microscope and spatial resolution of the camera. We found that the quality of the used lenses is high enough and the lenses are suitable for imaging with 1 microns spatial resolution with $8 \mathrm{keV} \mathrm{X}$-rays.

\section{ACKNOWLEDGMENTS}

We acknowledge the support provided by the Ministry of Education of the Czech Republic, project ICDMP/08 INGO Research in the International Centre for Dense Magnetized Plasma. This work was also partly supported by the Fund for Fundamental Investigations of Belarus.

\section{REFERENCES}

1. A. Snigirev, V. Kohn, I. Snigireva and B. Lengeler, "A Compound Refractive Lens for Focusing High-Energy Xrays", Nature (London) 384, 49 (1996).

2. Toshihisa Tomie, US Patent No. 5,594,773 (14 Jan. 1997) Foreign Appl. Date Feb. 18, 1994, Japan No. 6-45288. 
3. M. A. Piestrup, R. H. Pantell, J. T. Cremer and H. R. Beguiristain, "Compound Refractive Lens for X-rays," US Patent, 6,269,145 B1, allowed Jul. 31, 2001.

4. B. Lengeler, C.G. Schroer, B. Benner, T.F. Gunzler, M. Kuhlmann, J. Tummler, A.S. Simionovici, M. Drakopoulos, A. Snigirev, I. Snigireva, "Parabolic refractive X-ray lenses: a breakthrough in X-ray optics", Nucl. Instr. Meth. A, 467-468, 944 (2001).

5. V. Nazmov, L. Shabel'nikov, F. -J. Pantenburg, J. Mohr, E. Reznikova, A. Snigirev, I. Snigireva, S. Kouznetsov and M. DiMichiel, "Kinoform X-ray lens creation in polymer materials by deep X-ray lithography", Nucl. Instr. Meth. B 217, 409 (2004).

6. B. Nohammer, J. Hoszowska, A. K. Freund, C. David, "Diamond planar refractive lenses for third- and fourthgeneration X-ray sources", J. Synchrotron Rad., 10168 (2003).

7. Yu.I.Dudchik, N.N.Kolchevsky, “A microcapillary lens for X-rays”, Nucl. Instr. Meth. A 421, 361 (1999).

8. Yu.I.Dudchik, N.N.Kolchevsky, F.F.Komarov,Y.Kohmura, M.Awaji, Y.Suzuki, T.Ishikawa, "Glass capillary X-ray lens: fabrication technique and ray tracing calculations" Nucl.Instr.Meth. A 454, 512 (2000).

9. Yu. I. Dudchik, N.N. Kolchevsky, "Fabrication technique and ray tracing calculations for microcapillary x-ray lens", SPIE Proceedings. Advances in X-Ray Optics, 4145, 235 (2001).

10. Yu.I.Dudchik, N.N.Kolchevsky, F.F.Komarov, M.A.Piestrup, J.T.Cremer, C.K.Gary, R.H.Pantell, "Short-focallength compound refractive lenses for X-rays", Proceedings of SPIE. Fourth Generation X-Ray Sources and Ultrafast X-Ray Detectors, 5194, 56 (2004).

11. Yu.I.Dudchik, N.N.Kolchevsky, F.F.Komarov, M.A.Piestrup, J.T.Cremer, C.K.Gary H.Park and A.M.Khounsary, "Microspot X-ray focusing using short-focal length compound lenses", Rev. Sci. Instr. 75(11), 4651 (2004).

12. M.A.Piestrup, C.K.Gary, H.Park, J.L.Harris, J.T.Cremer, R.H.Pantell, Yu.I.Dudchik, N.N.Kolchevsky, F.F.Komarov, "Microscope using an X-ray tube and a bubble compound refractive lens", Appl. Phys. Letters, 86, 131104-1(2005).

13. Yu.I. Dudchik , F.F. Komarov, M.A. Piestrup, C.K. Gary, H. Park, J.T. Cremer, "Using of a microcapillary refractive X-ray lens for focusing and imaging”, Spectrochimica Acta Part B, 62, 598 (2007).

14. Yu.I. Dudchik, C.K. Gary, H. Park, R.H. Pantell, M.A. Piestrup, "Projection-type X-ray microscope based on a spherical compound refractive X-ray lens", Proceedings of SPIE. Advances in X-Ray/EUV Optics and Components II, 6705, 670509-1 (2007).

15. R. H. Pantell, J. Feinstein, H. R. Beguiristain, M. A. Piestrup, C. K. Gary and J. T. Cremer, "Characteristic of the thick compound refractive lens", Applied Optics 42, 719 (2003). 